\title{
Survival Reassessment at Tumor Recurrence in Soft Matter
}

\author{
Irina Trifonova ${ }^{1}$, Stefan Z. Stefanov ${ }^{2}$ \\ ${ }^{1}$ Specialized Hospital for Active Treatment in Oncology, Sofia, Bulgaria \\ ${ }^{2}$ Electricity System Operator (ESO EAD), Sofia, Bulgaria \\ Email: itrifa@abv.bg, stefanovsz@abv.bg
}

How to cite this paper: Trifonova, I. and Stefanov, S.Z. (2022) Survival Reassessment at Tumor Recurrence in Soft Matter. Open Journal of Modelling and Simulation, 10, 58-69.

https://doi.org/10.4236/ojmsi.2022.101004

Received: December 15, 2021

Accepted: January 14, 2022

Published: January 17, 2022

Copyright $\odot 2022$ by author(s) and Scientific Research Publishing Inc. This work is licensed under the Creative Commons Attribution International License (CC BY 4.0).

http://creativecommons.org/licenses/by/4.0/

(c) (i) Open Access

\begin{abstract}
The paper reassesses a survival at tumor recurrence in soft matter. First, the stability of structural motifs under shear in clusters of dipolar spheres is characterized. Next, there are introduced transitions between polymer knots and rhythms of these transitions are obtained. The sensor is built for these rhythms. Treatment, with a tensile force protocol, is modeled, when the tumor in soft matter is observed by the above sensor. Survival probability, at tumor recurrence in soft matter, is defined for the treatment with a tensile force protocol. It is stated that the survival probability at a tensile force protocol treatment in soft matter confirms or specifies the prognostic survival of 32 patients with breast cancer.
\end{abstract}

\section{Keywords}

Survival, Tumor Recurrence, Soft Matter, Polymer Knots, Vibrations, Time Crystal, Ultraweak Photon Emission

\section{Introduction}

Cancer is a multi-step process, where normal cells become transformed, grow, and may disseminate to establish new lesions in the body [1]. The physical properties of individual cells and of the tissue microenvironments are potent determinants of cancer progression. Cell and tissue mechanics, morphology, and migration are physical traits, linked to gene expression changes, which affect metastatic seeding, organ selectivity, and tumor growth [1] [2].

In the tumor microenvironment, some mechanical forces (shear, compression, tensile, etc.) promote tumor progression and treatment resistance [3]. As well, in a tumor microenvironment, there is physical energy, enabling for: 1) intracellular signaling by molecular vibration [4] [5];2) interplay between the circadian 
clock and cancer [6];3) rescue of damaged tissue [7].

Gene expression patterns and protein oscillation orchestrated by cancer stemness networks lead to state-space attractor cancer dynamics [8].

The paper looks for reassessment at tumor recurrence survival in soft matter. Here the tumor recurrence is understood as complex tumor dynamics in soft matter, modeling the upper physical qualities of individual cells and tissue microenvironment. In this case, the tumor in soft matter is defined as a structural motif, and the cyclic chemotherapy in soft matter, after which tumor recurrence occurs, is set as the restoration time.

\section{Stability of Structural Motifs under Shear}

In (DeVille et al., 2011) [9] is explicitly evaluated the stability of a specific stochastic two-dimensional non-Hamiltonian system, driven by additive white noise. The largest Lyapunov exponent is numerically computed, depending on contraction rate and shear. This dependence is represented [9] as a stability map.

In (Miller and Wales, 2005) [10] are found new energetically optimal structural motifs in clusters of dipolar spheres. These motifs are knots, links and coils. Morphology of these clusters is obtained depending on dipole strength and a cluster size.

Let the complexity of the above shear action coincides with the complexity of the considered morphology. Then a stability map and a structural map can be brought into coincidence through the following correspondence:

$$
\mu_{h}=2.25 \mu_{s}+1, \sigma=0.3636 N-2.1818
$$

Here the stability map is with a contraction rate $\mu_{h}$ and a shear $\sigma$. Also the structural map is with a dipole strength $\mu_{s}$ and a number of particles in a cluster $N$.

Since a structural map defines structural motifs in a tumor in soft matter, then the above bringing into coincidence allows finding the largest Lyapunov exponent on a linear scale for the structural motif in a tumor in soft matter:

$$
\lambda=-1.3221 \mu_{1}+0.8456, \mu_{1}=\mu_{s} \cos \left(21^{\circ}\right) .
$$

In Equation (2) $\mu_{s}$ is dipole strength of the structural motif in a tumor in soft matter.

Let the tumor in soft matter is with a structural motif with the largest Lyapunov exponent $\lambda$. As well, let $\lambda=-0.24$, when $\lambda$ belongs to the interval $-0.24<\lambda \leq 0$ and $\lambda=0.24$, when $\lambda$ belongs to the interval $0<\lambda<0.24$.

The tumor structural motifs in soft matter are with the largest Lyapunov exponent less or equal to $1.56, \lambda \leq 1.56$. Then the tumor structural motif in soft matter is defined from the corrected largest Lyapunov exponent $\lambda^{*}$, $\lambda^{*}=\lambda-1.56$.

\section{Transitions between Polymer Knots}

Closed polymer rings with nontrivial topological properties appear as DNA knots 
of living organisms [11]. The mechanical properties of polymer knots under stretching in a bad or good solvent are investigated in (Zhao and Ferrari, 2017) [11] by applying a given tensile force to a point of the knot while keeping another point fixed. Energy of the polymer conformation depends on [11] the tensile force, the number of contacts of the knot and the distance between the application point of the force and the origin. In (Zhao and Ferrari, 2017) [11], the mechanical properties of the short polymer knots under stretching by constant tensile force directed along $\mathrm{z}$-axis are studied on the simple cubic lattice.

Reference [11] shows a diagram with areas of accessible combinations of the number of contacts and the distance between the application point of the force and the origin for the unknot $0_{1}$, trefoil knot $3_{1}$ and the cinquefoil knot $5_{1}$, when all these knots are with a length of fifty lattice units.

According to this diagram, the boundary of the area of accessible combinations $\left(m_{1}, d_{1}\right)$ of the number of contacts $m_{1}$ and the distance $d_{1}$ between the application point of the force and the origin of the unknot $0_{1}$ can be approximated by an ellipse with a semi-major axe $a_{1}, a_{1}=61.77980$, a semi-minor axe $b_{1}$, $b_{1}=38.98505$, and a center $\left(m_{c, 1}, d_{c, 1}\right)=(5.37812,-0.14108)$.

According to this diagram, the boundary of the area of accessible combinations $\left(m_{2}, d_{2}\right)$ of the number of contacts $m_{2}$ and the distance $d_{2}$ between the application point of the force and the origin of the trefoil knot $3_{1}$ can be approximated by an ellipse with a semi-major axe $a_{2}, a_{2}=35.40223$, a semi-minor axe $b_{2}, \quad b_{2}=23.33651$, and a center $\left(m_{c, 2}, d_{c, 2}\right)=(20.90221,-0.04570)$.

According to this diagram, the boundary of the area of accessible combinations $\left(m_{3}, d_{3}\right)$ of the number of contacts $m_{3}$ and the distance $d_{3}$ between the application point of the force and the origin of the cinquefoil knot $5_{1}$ can be approximated by an ellipse with a semi-major axe $a_{3}, a_{3}=22.9614$, a semi-minor axe $b_{3}, b_{3}=17.36799$, and a center $\left(m_{c, 3}, d_{c, 3}\right)=(31.93548,-0.15345)$.

Let the tumor recurrence in soft matter is a transition from one polymer knot to another one (knot-knot transition), characterized by unstable oscillations of the number of contacts and of the distance between the application point of the force and the origin for each of the two knots, and with a cubic nonlinear interaction of those two oscillations. Let these transitions between polymer knots are: 1) $0_{1} \rightarrow 3_{1}$; 2) $0_{1} \rightarrow 5_{1}$; 3) $3_{1} \rightarrow 5_{1}$.

Let the treatment of tumor recurrence in soft matter is a state feedback control, which control stabilizes [12] the system of these two unstable oscillating processes in the neighborhood of the boundary of ellipsoidal manifold in $\mathbf{R}^{4}$, while distributing the energy of the transition between the two nonlinear processes.

Then the treatment at tumor recurrence in soft matter stabilizes one of the three transitions from a polymer knot to other via the feedback by (Gorobtsov et al., 2009) [12].

Oscillation frequencies of both stable limit cycles are [12]:

$$
\text { 1) } 0_{1} \rightarrow 3_{1}: \omega_{1}^{2}=b_{1}^{2} / a_{1}^{2}, \omega_{2}^{2}=b_{2}^{2} / a_{2}^{2} \text {; }
$$




\section{3) $3_{1} \rightarrow 5_{1}: \omega_{1}^{2}=b_{2}^{2} / a_{2}^{2}, \omega_{2}^{2}=b_{3}^{2} / a_{3}^{2}$.}

\section{Rhythms of Transitions}

Cancer-microenvironment interactions may consist [13] of chaotic gene expressions and a turbulent protein flow during a cancer pattern formation. The appearance of a turbulent flow is due to [14] a destruction of the laminar flow, while the laminar flow contains vorticity and potential components in the local zone of destruction.

Then let the angular velocity $\omega_{3, i}, i=1,2,3$, of the $i^{\text {th }}$ a knot-knot transition at tumor recurrence treatment in soft matter is [14]:

$$
\omega_{3, i}=v_{3}+8\left(v_{1}-\omega_{1, i}\right)\left(v_{3}+\omega_{1, i}\right)\left(v_{2}+\omega_{2, i}\right) /\left(\left(v_{2}-\omega_{2, i}\right)\left(v_{1}+\omega_{1, i}\right)\right), i=1,2,3 .
$$

Here $v_{1}$ and $\omega_{1, i}$ are, correspondingly, shear linear velocity and shear angular velocity of the flow for the $i$-th transition, $v_{2}$ and $\omega_{2, i}$ are, correspondingly, tsunami linear velocity and tsunami angular velocity of the flow for the $i^{\text {th }}$ transition, and $v_{3}$ is the restoration velocity from the initial tumor, $v_{3}=20 / t_{r}$. Here $t_{r}$ is the restoration time at cyclic chemotherapy in soft matter.

Knot-knot transition is an open thermodynamic system at the observed treatment. This system is with nonequilibrium dynamics, due to dissipation and vorticity at this treatment. According to quantum thermodynamics [15], the internal energy becomes extensive after the system reaches equilibrium. At the studied treatment, equilibration refers to tumor recurrence in soft matter from $\$ 3$, evolving to steady self-oscillations. At a knot-knot transition, this extensive property depends [15] on the oscillation frequencies $\omega_{1}^{2}$ and $\omega_{2}^{2}$ from Equation (3) and is fulfilled $\omega_{1, i}=\omega_{1}^{2}, \omega_{2, i}=\omega_{2}^{2}$.

Let a knot-knot transition, at the observed treatment, is entanglement propagation following a local quench in $2+1$ dimensional holographic conformal field theories. Then [16], the entanglement velocity is fairly close to hydrodynamical velocity $v_{\text {shear, }} v_{\text {shear }}=0.665$, related to entropy production. Also [16], the tsunami velocity, $v_{\text {tsunami }}=0.687$, which appears as a lower bound of entanglement velocity, is a lower bound on information processing. Therefore, it is assumed that $v_{1}=v_{\text {shear }}, v_{2}=v_{\text {tsunami }}$.

Time of the $i$-th knot-knot transition is:

$$
T_{i}=2 \pi / \omega_{3, i}, i=1,2,3 .
$$

Knot-knot rhythms of transitions are the times $10 T_{i}, i=1,2,3$, which are found via entanglement spectroscopy [17] of this transition.

\section{Tensile Force Protocol for Treatment}

A portion of dark energy contained in a five-dimensional universe, entangled with Brans-Dicke field, can be used for human beings treatment [18]. Therefore, this portion of dark energy should be transformed [18] into other forms of energy.

Let the treatment energy, at each of the three transitions, is determined through 
the part of dark energy that can serve for treatment [18], in the following way [14]:

$$
\begin{aligned}
& \text { 1) } \left.\left.0_{1} \rightarrow 3_{1}: E_{0} \rightarrow E_{3}, 2\right) 0_{1} \rightarrow 5_{1}: E_{0} \rightarrow E_{5}, 3\right) 3_{1} \rightarrow 5_{1}: E_{3} \rightarrow E_{5} \text {, } \\
& E_{0}=(3 /(4 \pi)) \exp \left(\lambda_{1}\left(5 T_{2} / 20\right)\right)\left(\lambda_{1}-12-36 / \lambda_{1}^{2}\right), \\
& E_{3}=E_{r 1} \exp \left(a b s\left(T_{2}-2 T_{1}\right)\left(\ln \left(\left(a b s\left(T_{2}-2 T_{1}\right)\right)^{1 / 2}\right)-0.5\right)\right) \text {, } \\
& E_{r 1}=(3 /(4 \pi)) \exp \left(\lambda_{1}\left(10 T_{1} / 20\right)\right)\left(\lambda_{1}-12-36 / \lambda_{1}^{2}\right) \text {, } \\
& E_{5}=E_{r 3} \exp \left(\operatorname{abs}\left(T_{2}-T_{3}\right)\left(\ln \left(\left(a b s\left(T_{2}-T_{3}\right)\right)^{1 / 2}\right)-0.5\right)\right), \\
& E_{r 3}=(3 /(4 \pi)) \exp \left(\lambda_{1}\left(5 T_{3} / 20\right)\right)\left(\lambda_{1}-12-36 / \lambda_{1}^{2}\right) \text {, } \\
& \lambda_{1}=-\lambda^{*} / 5+0.188 \text {. }
\end{aligned}
$$

Let the transition energy $\left(0_{1} \rightarrow 5_{1}\right)$ that serves for tumor treatment in soft matter, is the specific energy of the unknot $0_{1}$ with a length of 50 lattice units, $(\langle E\rangle / 50)\left(0_{1}\right)=E_{0}$, for $E_{0}$ from Equation (6). Then, from the graphic of the specific energy of the unknot $0_{1}$ with length of 50 lattice units under stretching in bad solvent at high temperature [11] is found the tensile force $F_{0}$, corresponding to $E_{0}$. Next, from the graphic of the heat capacity of the unknot $0_{1}$ with length of 50 lattice units under stretching in bad solvent at high temperature [11], $(C / 50)\left(0_{1}\right)$, is found the heat capacity $C_{0}$, corresponding to the tensile force $F_{0}$.

Let the transition energy $\left(0_{1} \rightarrow 3_{1}\right)$ that serves for tumor treatment in soft matter, is the specific energy of the trefoil knot $3_{1}$ with a length of 50 lattice units, $(\langle E\rangle / 50)\left(3_{1}\right)=E_{3}$, for $E_{3}$ from Equation (6). Then, from the graphic of the specific energy of the trefoil knot $3_{1}$ with length of 50 lattice units under stretching in bad solvent at high temperature [11] is found the tensile force $F_{3}$, corresponding to $E_{3}$. Next, from the graphic of the heat capacity of the trefoil knot $3_{1}$ with length of 50 lattice units under stretching in bad solvent at high temperature [11], $(C / 50)\left(3_{1}\right)$, is found the heat capacity $C_{3}$, corresponding to the tensile force $F_{3}$.

Let the transition energy $\left(3_{1} \rightarrow 5_{1}\right)$ that serves for tumor treatment in soft matter is the specific energy of the cinquefoil knot $5_{1}$ with a length of 50 lattice units, $(\langle E\rangle / 50)\left(5_{1}\right)=E_{5}$, for $E_{5}$ from Equation (6). Then from the graphic of the specific energy of the cinquefoil knot $5_{1}$ with a length of 50 lattice units under stretching in bad solvent at high temperature [11] is found the tensile force $F_{5}$, corresponding to $E_{5}$. Next, from the graphic of the heat capacity of the cinquefoil knot $5_{1}$ with length of 50 lattice units under stretching in bad solvent at high temperature [11], $(C / 50)\left(5_{1}\right)$, is found the heat capacity $C_{5}$, corresponding to the tensile force $F_{5}$.

Treatment protocol, at each of the three transitions, consists in simultaneously implying the following two tensile forces:

1) $0_{1} \rightarrow 3_{1}$ : Tensile force $F_{0}$ on the polymer knot $0_{1}$ and tensile force $F_{3}$ on the polymer knot $3_{1}$; 
2) $0_{1} \rightarrow 5_{1}$ : Tensile force $F_{0}$ on the polymer knot $0_{1}$ and tensile force $F_{5}$ on the polymer knot $5_{1}$;

3) $3_{1} \rightarrow 5_{1}$ : Tensile force $F_{3}$ on the polymer knot $3_{1}$ and tensile force $F_{5}$ on the polymer knot $5_{1}$.

\section{Sensor for Tumor in Soft Matter}

Let the recurrence tumor in soft matter is a rare event in driven diffusive media. Then it can be assumed that a packing field, which triggers condensation instability, triggers immune evasion. Therefore a continuous time crystal from rare event can be built [19] of the recurrence tumor in soft matter. This time crystal is a sensor for the recurrence tumor in soft matter.

Packing order parameter $R\left(\lambda_{p}\right)$ measures particles' coherent motion [19]. This parameter depends on intensity of the biasing field $\lambda_{p}$. Let this intensity of the biasing field $\lambda_{p}$ is equal to the largest Lyapunov's exponent $\lambda^{*}$ from $\$ 2, \lambda_{p}=\lambda^{*}$. Here is assumed that the intensive biasing field is conjugated to the extensive current in a way similar to the stability map relation from $\$ 2$ between contraction rate and shear. Then the packing order parameter $R\left(\lambda^{*}\right)$ of the recurrence tumor in soft matter is found from the graphic in (Hurtado-Gutiérrez et al., 2020) [19].

\section{Sensor's Tuning}

Let the tumor recurrence in soft matter is weakly measured through entanglement spectroscopy of knot-knot transition. Let the tuning of the above sensor is achieved by a knot-knot transition feedback.

Applying feedback and week measurements to the quantum system of a knotknot transition induces [20] phase transitions beyond the dissipative ones. The critical exponent of the knot-knot transition $\alpha(s)$ is found from the graphic [20] of the dependency of critical exponent $\alpha$ on feedback exponent $s, s=\lambda_{1}$. Here it is assumed that the exponent of the feedback depends linearly, as in Equation (6), from the corrected largest Lyapunov's exponent $\lambda^{*}$.

At controlling the critical knot-knot transition exponent, the feedback response of the transition for the times $T_{1}, T_{2}, T_{3}$ from Equation (5) is [20]:

$$
k_{1}=s\left(2 T_{1} / T_{2}\right)^{s+1}, k_{2}=s\left(T_{3} / T_{2}\right)^{s+1} .
$$

The tuning of the sensor for tumor recurrence in soft matter, via controlling the critical knot-knot transition exponent, is:

$$
R_{1}\left(\lambda^{*}\right)=(2 / \pi) k_{1} R\left(\lambda^{*}\right), R_{2}\left(\lambda^{*}\right)=(2 / \pi) k_{2} R\left(\lambda^{*}\right) .
$$

\section{Treatment Model with a Tensile Force Protocol}

Treatment at tumor recurrence in soft matter that stabilises knot-knot transitions via the feedback by (Gorobtsov et al., 2009) [12], is:

$$
\begin{gathered}
\mathrm{d} x_{1} / \mathrm{d} t=x_{2} \\
\mathrm{~d} x_{2} / \mathrm{d} t=\alpha_{1} x_{2}-\omega_{1}^{2} x_{1}-u_{1}
\end{gathered}
$$




$$
\begin{gathered}
\mathrm{d} x_{3} / \mathrm{d} t=x_{4} \\
\mathrm{~d} x_{4} / \mathrm{d} t=\alpha_{2} x_{4}-\omega_{2}^{2} x_{3}-u_{2} \\
u_{1}=\beta_{2,1} x_{2} x_{1}^{2}+\beta_{2,2} x_{2}^{3}+\beta_{2,3} x_{2} x_{3}^{2}+\beta_{2,4} x_{2} x_{4}^{2} \\
u_{2}=\beta_{4,3} x_{4} x_{3}^{2}+\beta_{4,4} x_{4}^{3}+\beta_{4,1} x_{4} x_{1}^{2}+\beta_{4,2} x_{4} x_{2}^{2} \\
\beta_{2,1}=\alpha_{1} / a_{10}^{2}, \beta_{2,2}=\alpha_{1} / b_{10}^{2}, \beta_{4,3}=\alpha_{2} / a_{20}^{2} \\
\beta_{4,4}=\alpha_{2} / b_{20}^{2}, \beta_{2,3}=\alpha_{1} / a_{20}^{2}, \beta_{4,1}=\alpha_{2} / a_{10}^{2} \\
b_{10}^{2} \beta_{4,2}+b_{20}^{2} \beta_{2,4}+b_{20}^{2} \alpha_{2}+b_{10}^{2} \alpha_{1}=0 .
\end{gathered}
$$

It is accepted here, that for each of the three transitions, is fulfilled:

$$
\begin{aligned}
& 0_{1} \rightarrow 3_{1}: x_{1}=\left(m_{1}-m_{c, 1}\right), x_{2}=\left(d_{1}-d_{c, 1}\right), x_{3}=\left(m_{2}-m_{c, 2}\right), \\
& x_{4}=\left(d_{2}-d_{c, 2}\right), a_{10}=a_{1}, b_{10}=b_{1}, a_{20}=a_{2}, b_{20}=b_{2} ; \\
&2) \begin{array}{l}
1 \\
0_{1} \rightarrow 5_{1}: x_{1}
\end{array}=\left(m_{1}-m_{c, 1}\right), x_{2}=\left(d_{1}-d_{c, 1}\right), x_{3}=\left(m_{3}-m_{c, 3}\right), \\
& x_{4}=\left(d_{3}-d_{c, 3}\right), a_{10}=a_{1}, b_{10}=b_{1}, a_{20}=a_{3}, b_{20}=b_{3} ; \\
& 3_{1} \rightarrow 5_{1}: x_{1}=\left(m_{2}-m_{c, 2}\right), x_{2}=\left(d_{2}-d_{c, 2}\right), x_{3}=\left(m_{3}-m_{c, 3}\right), \\
& x_{4}=\left(d_{3}-d_{c, 3}\right), a_{10}=a_{2}, b_{10}=b_{2}, a_{20}=a_{3}, b_{20}=b_{3} .
\end{aligned}
$$

Also $\omega_{1}$ and $\omega_{2}$ are oscillation frequencies for the two steady state self-oscillations, $\alpha_{1}$ and $\alpha_{2}$ characterize instability of the two oscillations, $\alpha_{1}>0, \alpha_{2}>0, u_{1}$ and $u_{2}$ are two state controls, $\beta_{i, j}$ are control coefficients. Oscillation frequencies $\omega_{1}$ and $\omega_{2}$ are determined in Equation (3).

In treatment model (9) (Model (9)), the distance between the application point of the force and the origin is considered to be due to the variation of the number of contacts for each of the two knots of a transition.

Interaction of both controls $u_{1}$ and $u_{2}$ is defined by the coefficients $\beta_{4,2}$ and $\beta_{2,4}$ from Model (9). At this treatment it is assumed that the coefficients $\beta_{4,2}$ and $\beta_{2,4}$ are:

$$
\beta_{4,2}=\alpha_{1}, \beta_{2,4}=-\alpha_{1}\left(2 b_{10}^{2} / b_{20}^{2}+1\right) .
$$

Then the constraint from Model (9) is fulfilled for these coefficients.

Treatment model at tumor recurrence in soft matter is with equal coefficients $\alpha_{1}$ and $\alpha_{2}$, because both oscillations are a part of one transition. Also let these coefficients be equal to square root of the corrected packing order parameter from $₫ 7:$ 1) $\quad \alpha_{1}=\alpha_{2}=R_{1}\left(\lambda^{*}\right)^{1 / 2}$;2) $\alpha_{1}=\alpha_{2}=R_{2}\left(\lambda^{*}\right)^{1 / 2}$.

At the first connection in the Model (9), the amplitudes of the two settled down self-oscillations $A_{11}$ and $A_{21}$ are [12]:

$$
A_{11}=a_{10}\left(x_{1}^{2} / a_{10}^{2}+x_{2}^{2} / b_{10}^{2}\right)^{1 / 2}, A_{21}=a_{20}\left(x_{3}^{2} / a_{20}^{2}+x_{4}^{2} / b_{20}^{2}\right)^{1 / 2} .
$$

At the second connection in the Model (9), the amplitudes of the two settled down self-oscillations $A_{12}$ and $A_{22}$ are [12]:

$$
A_{12}=a_{10}\left(x_{1}^{2} / a_{10}^{2}+x_{2}^{2} / b_{10}^{2}\right)^{1 / 2}, A_{22}=a_{20}\left(x_{3}^{2} / a_{20}^{2}+x_{4}^{2} / b_{20}^{2}\right)^{1 / 2} .
$$

Then the tumor recurrence treatment in soft matter is found by integrating 
the Model (9) in a time interval $\left[0, T_{f, i}\right]$ with an integration step $\Delta t_{i}=T_{f, i} / 20$, $i=1,2,3$, when this system is with the following initial state:

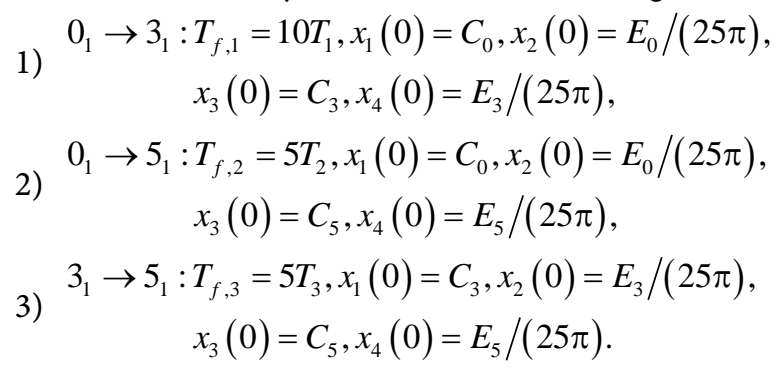

Here is assumed that the number of contacts in the initial moment is determined by the heat capacity and that the real distance between the application point of the force and the origin in the initial moment, is equal to the treatment energy, divided by the number of segments per knot $N, N=50$. Here the real distance between the application point of the force and the origin is the distance between the application point of the force and the origin, multiplied by the half of the size of the compact direction in the boundary theory $L_{c}$ by (Myers et al., 2017) [17], $L_{c}=\pi$.

In this treatment model it is possible the number of contacts to be negative. In this case, tumor in soft matter is considered as nanocluster, trapped in metastable state, differing from the true thermodynamic equilibrium state. This trapping appears [21] due to high-energy barriers in cluster phase space, separating the tumor in soft matter.

At this consideration the tumor in soft natter is a "small" system [21] in sense that the range of the relevant interaction is greater than the characteristic size of the system of nanoclusters. In this case the above treatment leads to a stable boundary cycle of a large interaction of the tumor in soft matter with environment, coexisting with a stable boundary cycle of a small decoherence between tumor in soft matter and environment. Then the above treatment looks like a rescue [22] from DNA damages of the p53 regulatory network of a cell population.

\section{Survival at Tensile Force Protocol Treatment}

Let a tumor recurrence in soft matter is a two level atom, with a level, excited by the treatment, and with a level, not excited by the treatment. Then the knot-knot transition at above treatment can be represented as a two level atom interacting with an optical cavity (near environment), presenting either dephasing or photon losses to a far environment. At that time decoherence of the optical cavity mimics the effects of far environment. Thus the knot-knot transition at the above treatment is modeled with a qubit coupled to sequentially nested environment by (Torres and Seligman, 2017) [23]. The above treatment protects the coherence of the knot-knot transition, while increases the coupling between near and far environments through a corrected packing order parameter.

Knot-knot transition at the above treatment can be detected through measurement of ultraweak photon emissions so as it is [24] at tumor detections. 
Let in the above model of the knot-knot transition a two level atom interacts with: 1) an optical cavity with strength $g=A_{21}\left(T_{f, i} / 2\right) / 10$ and the cavity presents dephasing at rate $\left.\gamma=A_{11}\left(T_{f, i} / 2\right) / 10 ; 2\right)$ an optical cavity with strength $g=A_{22}\left(T_{f, i} / 2\right) / 10$ and the cavity presents photon losses at rate $k=A_{12}\left(T_{f, i} / 2\right) / 10$ to a far environment. Then the survival probability until moment $10 T_{f, i} / 2, i=1,2,3$, at treatment with a tensile force protocol, is obtained as an atomic excitation probability $p_{e, 1}\left(T_{f, i} / 2\right), i=1,2,3$, from (Torres and Seligman, 2017) [23], depending on: 1) the ratio $\gamma / g=A_{11}\left(T_{f, i} / 2\right) / A_{21}\left(T_{f, i} / 2\right)$ or the ratio $\left.k / g=A_{12}\left(T_{f, i} / 2\right) / A_{22}\left(T_{f, i} / 2\right) ; 2\right)$ the detuning $\delta$. Here $\delta$ is [23] the detuning of the atomic transition frequency from the frequency of the mode.

Let the survival probability is found from: 1) a diagram for atomic excitation probability by (Torres and Seligman, 2017) [23], when the cavity presents dephasing to a far environment and a detuning is $\delta / g=0 ; 2$ ) a diagram for atomic excitation probability by (Torres and Seligman, 2017) [23], when the cavity presents the photon losses to a far environment and detuning is $\delta / g=0.8$.

Each of these two diagrams by (Torres and Seligman, 2017) [23], with a time in months, is similar to the diagram for a probability of distant metastasis-free, given in months, survival for patients with breast cancer by (Bianchini et al., 2010) [25].

Let the age of the patients is characterized by different detuning. Then it can be assumed that the survival probability at the re-treatment with a protocol for tensile force, found according the first diagram, is comparable to the survival probability for the middle-aged (45 - 59 age) patients and younger patients with breast cancer, and the probability survival, found according the second diagram is comparable to the survival probability for the elderly (60 - 74 age) and old patients with breast cancer.

\section{Results}

Database is used for 424 patients with breast cancer, who were under treatment at the Clinic of Chemotherapy, Specialized Hospital for Active Treatment in Oncology, Sofia, Bulgaria, throughout 2003-2014. A group of 32 patients is randomly selected from the 424 patients with different TNM staging ( $\mathrm{T}$ - tumor size, $\mathrm{N}-$ lymph node status, $\mathrm{M}$-distant metastasis), histology and immunohistochemical characteristics. All patients' proliferation index is assessed. The gene expression is not tested. Their medical history is retrospectively tracked, their current survival is reported (March 2020) and is investigated a correlation with the standard clinicopathological criteria of risk assessment: TNM staging, histology, tumor differentiation grade, (ER, PR, HER2) receptor status. Patients with soft tissue carcinoma and other carcinoma are not included in the group.

Survival probability at cyclic chemotherapy and the recovery time are obtained from the proliferation index of the concrete tumor via chemotherapy model in soft matter by (Trifonova et al., 2014) [26]. Structural motif of each of these tumors is related to a structural motif by (Miller and Wales, 2005) [10]. 
Survival probability at cyclic chemotherapy in soft matter by (Trifonova et al., 2014) [26], is indeterminate for five patients. These cases remain outside from the current consideration.

Survival probability at a treatment with a tensile force protocol is found for 27 patients. It is assumed that survival probability at a treatment with a tensile force protocol is changed slightly compared to the survival probability at cyclic chemotherapy by (Trifonova et al., 2014) [26], when the absolute value of this change does not exceed 0.05 in close moments of time. Also, it is assumed that the survival probability, at a treatment with a tensile force protocol, changes a lot, compared to the success of cyclic chemotherapy by (Trifonova et al., 2014) [26], when the absolute value of this change exceeds 0.05 in close moments of time. This change is uncertain in four cases due to a large discrepancy between the timing of the two treatments.

Survival reassessment, at a tumor recurrence in soft matter, is found by comparing the survival probability at a treatment with a tensile force protocol for a tumor recurrence in soft matter, and the survival probability at cyclic chemotherapy of the initial tumor development in soft matter. The actual survival is then analyzed as the actual data are taken from the patients' medical history.

Treatment with a tensile force protocol improves the survival assessment in eleven cases (48\%). Great assessment improvement is achieved in seven of these cases at tumor treatment $\left(0_{1} \rightarrow 5_{1}\right)$ in soft matter, regardless of the patients' age. In the rest four cases assessment improvement at tumor treatment $\left(0_{1} \rightarrow 3_{1}\right)$ in soft matter is achieved. In these eleven cases the specification of the prognostic survival is achieved, so that it corresponds to the actual one.

Treatment with a tensile force protocol keeps on the survival assessment in eight cases (35\%) of middle-aged and younger patients. In these cases the prognostic survival is confirmed.

Treatment with a tensile force protocol worsens the survival assessment in four cases (17\%). These cases are with obscure structural motif.

\section{Conclusions}

Survival reassessment, of a tumor recurrence in soft matter, is done in this paper:

1) Stability of structural motifs under shear in clusters of dipolar spheres, is characterized via Lyapunov exponent;

2) Transitions between polymer knots are introduced, when these knots are exposed to stretching, and a transition occurs as interconnected vibrations;

3) Rhythms of transitions are obtained through entanglement spectroscopy;

4) A tensile force protocol for treatment is proposed, when the energy of this treatment is equal to the part of dark energy that can serve for treatment;

5) Sensor for a tumor in soft matter, as a time crystal for discovering a rare phenomenon, is built;

6) The sensor for a tumor in soft matter, via the critical exponent of the tran- 
sitions between polymer knots, is tuned;

7) A treatment with a tensile force protocol, as vibration isolation, is modeled;

8) Survival probability, as atomic excitation probability at ultraweak photon emission, is given.

Survival reassessment, at tumor recurrence in soft matter, confirms or specifies the prognostic survival of 32 patients with breast cancer at a cyclic chemotherapy model in soft matter. This shows that the prognostic survival of patients with the breast cancer can be assessed by this model.

\section{Conflicts of Interest}

The authors declare no conflicts of interest regarding the publication of this paper.

\section{References}

[1] So, W.Y. and Tanner, K. (2021) Emerging Principles of Cancer Biophysics. Faculty Reviews, 10, Article No. 61. https://doi.org/10.12703/r/10-61

[2] Nishida-Aoki, N. and Gujral, T.S. (2019) Emerging Approaches to Study Cell-Cell Interactions in Tumor Microenvironment. Oncotarget, 10, 785-797. https://doi.org/10.18632/oncotarget.26585

[3] Stylianopoulos, T., Munn, L.L. and Jain, R.K. (2018) Reengineering the Physical Microenvironment of Tumors to Improve Drug Delivery and Efficiency: From Mathematical Modeling to Bench to Bedside. Trends in Cancer, 4, 292-319. https://doi.org/10.1016/j.trecan.2018.02.005

[4] Jaross, W. (2020) The Possible Role of Molecular Vibration in Intracellular Signaling. Journal of Cellular Signaling, 1, 180-186. https://doi.org/10.33696/Signaling.1.027

[5] Funk, R.H.W. (2018) Biophysical Mechanisms Complementing "Classical” Cell Biology. Frontiers in Bioscience-Landmark, 23, 921-939. https://doi.org/10.2741/4625

[6] Sulli, G., Lam, M.T.Y. and Panda, S. (2019) Interplay between Circadian Clock and Cancer: New Frontiers for Cancer Treatment. Trends in Cancer, 5, 475-494. https://doi.org/10.1016/j.trecan.2019.07.002

[7] Facchin, F., Canaider, S., Tassinari, R., Zannini, C., Bianconi, E., Taglioli, V., et al. (2019) Physical Energies to the Rescue of Damaged Tissues. World Journal of Stem Cells, 11, 297-321. https://doi.org/10.4252/wjsc.v11.i6.297

[8] Uthamacumaran, A. (2021) A Review of Dynamical Systems Approaches for the Detection of Chaotic Attractors in Cancer Networks. Patterns, 2, Article ID: 100226. https://doi.org/10.1016/j.patter.2021.100226

[9] DeVille, R.L., Namachchivaya N.S. and Rapti Z. (2011) Stability of a Stochastic TwoDimensional Non-Hamiltonian System. SIAM Journal on Applied Mathematics, 71, 1458-1475. https://doi.org/10.1137/100782139

[10] Miller, M.A. and Wales, D.J. (2005) Novel Structural Motifs in Clusters of Dipolar Spheres: Knots, Links and Coils. Journal of Physical Chemistry B, 109, 23109-231112. https://doi.org/10.1021/jp0549632

[11] Zhao, Y. and Ferrari, F. (2017) The Topological Effect on the Mechanical Properties of Polymer Knots. Physica A: Statistical Mechanics and Its Applications, 486, 44-64. https://doi.org/10.1016/j.physa.2017.05.015

[12] Gorobtsov, A.S., Grigor'eva, O.E. and Ryzhov, E.N. (2009) Attraction Ellipsoids and 
Design of Non-Linear Oscillation Modes. Automation and Remote Control, 70, 13011308. (in Russian)

[13] Uthamacumaran, A. (2020) Cancer: A Turbulence Problem. Neoplasia, 22, 759-769. https://doi.org/10.1016/j.neo.2020.09.008

[14] Panchenkov, A.N. (2006) Complex Spaces in Hydrodynamics: Complex Navier-Stokes Equations. arXiv: physics/0609159.

[15] Hsiang, J.T., Chou, C.H., Subaşi, Y. and Hu, B.L. (2018) Quantum Thermodynamics from the Nonequilibrium Dynamics of Open Systems: Energy, Heat Capacity and the Third Law. Physical Review E, 97, Article ID: 012135. https://doi.org/10.1103/PhysRevE.97.012135

[16] Rozali, M. and Vincart-Emard, A. (2017) Comments on Entanglement Propagation. Journal of High Energy Physics, 2017, Article No. 44. https://doi.org/10.1007/JHEP06(2017)044

[17] Myers, R.C., Rozali, M. and Way, B. (2017) Holographic Quenches in a Confined Phase. Journal of Physics A: Mathematical and Theoretical, 50, Article ID: 494002. https://doi.org/10.1088/1751-8121/aa927c

[18] Singh, K.M. and Singh, K.P. (2019) Whether Dark Energy Can Contribute to the Treatment and Healing of Diseases. Modern Physics Letters A, 34, Article ID: 1950260. https://doi.org/10.1142/S0217732319502602

[19] Hurtado-Gutiérrez, R., Carollo, F., Pérez-Espigares, C. and Hurtado, P.I. (2020) Building Continuous Time Crystals from Rare Events. Physical Review Letters, 125, Article ID: 160601. https://doi.org/10.1103/PhysRevLett.125.160601

[20] Ivanov, D.A., Ivanova, T.Y., Caballero-Benitez, S.F. and Mekhov, I.B. (2020) FeedbackInduced Quantum Phase Transitions Using Weak Measurements. Physical Review Letters, 124, Article ID: 010603. https://doi.org/10.1103/PhysRevLett.124.010603

[21] Michaelian, K. and Santamaría-Holek, I. (2007) Critical Analysis of Negative Heat Capacity in Nanoclusters. EPL, 79, Article No. 43001. https://doi.org/10.1209/0295-5075/79/43001

[22] Jiang, C., Zhang, Y. and Wang, W. (2021) Codimension-3 Bifurcation in the p53 Regulatory Network Model. International Journal of Bifurcation and Chaos, 31, Article ID: 2150104. https://doi.org/10.1142/S0218127421501042

[23] Torres, J.M. and Seligman, T.H. (2017) Protecting Coherence by Environmental Decoherence: A Solvable Paradigmatic Model. New Journal of Physics, 19, Article ID: 113016. https://doi.org/10.1088/1367-2630/aa910c

[24] Murugan, N.J., Persinger, M.A., Karbowski, L.M. and Dotta, B.T. (2020) Ultraweak Photon Emissions as a Non-Invasive, Early-Malignancy Detection Tool: An In Vitro and In Vivo Study. Cancers, 12, Article No. 1001. https://doi.org/10.3390/cancers 12041001

[25] Bianchini, G., Iwamoto, T., Qi, Y., Charles, C., Shiang, C.Y., Wang, B., et al. (2010) Prognostic and Therapeutic Implications of Distinct Kinase Expression Patterns in Different Subtypes of Breast Cancer. Cancer Research, 70, 8852-8862. https://doi.org/10.1158/0008-5472.CAN-10-1039

[26] Trifonova, I., Kurteva, G. and Stefanov, S.Z. (2014) Success of Chemotherapy in Soft Matter. https://arxiv.org/pdf/1404.0936 